\title{
Silencing of peroxiredoxin II by promoter methylation is necessary for the survival and migration of gastric cancer cells
}

\author{
Soo Hyun Hong ${ }^{1,6}$, Chengchun Min ${ }^{1,6}$, Yukyung Jun ${ }^{1,6}$, Doo Jae Lee ${ }^{1,2}$, Seung Hwa Kim ${ }^{3}$, Joo Hyun Park ${ }^{4}$, \\ Jae Ho Cheong ${ }^{3}$, Yoon Jung Park ${ }^{4}$, Soo-Youl Kim ${ }^{5}$, Sanghyuk Lee ${ }^{1,2}$ and Sang Won Kang ${ }^{1,2}$ \\ Peroxiredoxin (Prx), a family of ubiquitous thiol peroxidases, functions as a redox signaling regulator that controls cellular $\mathrm{H}_{2} \mathrm{O}_{2}$ in \\ mammalian cells and has recently received attention for being overexpressed in various cancer types. In this study, we show that \\ Prx type II (PrxII) is rather silenced in gastric cancer cells. Prxll expression is severely downregulated in 9 out of the 28 gastric \\ cancer cell lines. Strikingly, Prxll expression is completely lost in three cell lines, MKN28, MKN74 and SNU484. Loss of Prxll \\ expression is due to DNA methyltransferase 1-dependent methylation at the promoter region of the Prxll gene. Restoration of Prxll \\ expression using a retroviral system markedly reduces the colony-forming ability and migratory activity of both MKN28 and \\ SNU484 cells by inhibiting Src kinase. Mechanistically, Prxll peroxidase activity is essential for regulating gastric cancer cell \\ migration. Bioinformatics analysis from The Cancer Genome Atlas stomach cancer data (STAD) revealed significantly low Prxll \\ expression in gastric cancer patients and a negative correlation between Prxll expression and methylation levels. More importantly, \\ low Prxll expression also strongly correlates with poor survival in cancer patients. Thus our study suggests that Prxll may be the \\ first thiol peroxidase that simultaneously regulates both survival and metastasis in gastric cancer cells with high clinical relevance. \\ Experimental \& Molecular Medicine (2018) 50, e443; doi:10.1038/emm.2017.267; published online 9 February 2018
}

\section{INTRODUCTION}

Gastric cancer is a leading cause of death worldwide, accounting for nearly 1000000 new cases annually and $>700000$ deaths in 2014. Moreover, an estimation of 26370 diagnosed cases of gastric cancer and eventually 10730 deaths were reported in the United States in 2016. ${ }^{1,2}$ In most cases, gastric cancer treatment relies on gastrectomy and chemotherapy. However, due to heterogeneity, gastric cancer recurrence rates are relatively high, and re-resection is no longer an available option for patients with metastatic cases. ${ }^{3}$ Therefore, therapeutics targeting gastric cancer cell metastasis are of clinical significance.

Reactive oxygen species (ROS), including hydrogen peroxide $\left(\mathrm{H}_{2} \mathrm{O}_{2}\right)$ and superoxide anion $\left(\mathrm{O}^{2-}\right)$, are generated during cellular metabolism. Excess amounts of ROS damage cellular macromolecules, such as proteins, DNA and membrane lipids. ${ }^{4}$ However, recently accumulating evidence indicates that a transient increase in $\mathrm{H}_{2} \mathrm{O}_{2}$ has a signaling messenger role in cell proliferation and differentiation, for instance, by reversibly inactivating cysteine residues in protein tyrosine phosphatases. ${ }^{5,6}$ Under normal conditions, ROS levels are homeostatically maintained by cellular antioxidant enzymes, such as catalase, glutathione peroxidase (GPx) and peroxiredoxin (Prx), which are localized in various cellular compartments. In particular, Prx is the latest family of antioxidant enzymes that was initially identified in Saccharomyces cerevisiae as a protein-protecting glutamine synthetase against mixedfunction oxidation systems. ${ }^{7}$ It was later discovered that Prx contains conserved cysteine residues in its active site and exhibits $\mathrm{H}_{2} \mathrm{O}_{2}$-reducing peroxidase activity by coupling exclusively with thioredoxin, thioredoxin reductase and NADPH. ${ }^{8}$ The main role of Prx in mammalian cells has long been believed to be as an antioxidant system that scavenges for excess cellular ROS. However, more evidence from recent studies has suggested that Prx has regulatory roles in diverse cancer cell activities, such as proliferation, migration and

\footnotetext{
${ }^{1}$ Department of Life Sciences, Ewha Womans University, Seoul, Korea; ${ }^{2}$ Research Center for Cell Homeostasis, Ewha Womans University, Seoul, Korea; ${ }^{3}$ Department of Surgery, Yonsei University College of Medicine, Seoul, Korea; ${ }^{4}$ Department of Nutritional Science and Food Management, Ewha Womans University, Seoul, Korea and ${ }^{5}$ Division of Cancer Biology, Research Institute, National Cancer Center, Goyang, Korea

${ }^{6}$ These authors contributed equally to this work.

Correspondence: Professor SW Kang, Department of Life Sciences, Ewha Womans University Science Building C/Room 504, 52 Ewhayeodae-gil, Seodaemun-gu, Seoul 120-750, Korea.

E-mail: kangsw@ewha.ac.kr

Received 1 May 2017; revised 4 September 2017; accepted 13 September 2017
} 
programmed cell death. ${ }^{9-11}$ In relation to such novel cellular functions, the regulation of Prx activity or expression has been receiving great attention. We and others have shown that Prx activity is tightly regulated by posttranslational modifications, such as phosphorylation, acetylation and hyperoxidation. ${ }^{12}$ In addition to modification, PrxI is transcriptionally regulated by Nrf2. ${ }^{13}$ In contrast, PrxII expression has been shown to be regulated by aberrant promoter methylation in some cancer types, such as leukemia, lymphoma and melanoma. ${ }^{11,14,15}$ In relation to gastric cancer, promoter methylation of the GPx1 and GPx3 genes has been reported in the past without functional relevance. ${ }^{16,17}$ However, aberrant promoter methylation of PrxII associated with gastric cancer has never been investigated.

As a stable repressive mark, DNA methylation involves the covalent transfer of a methyl group to the fifth carbon of a cytosine, resulting in gene silencing. Although DNA methylation has a crucial role in normal developmental processes, tumorigenesis arises when tumor-suppressor gene promoters are repressed by hypermethylation or the loss of global methylation. ${ }^{18,19}$ DNA methylation is catalyzed by a family of DNA methyltransferases (DNMTs), which contain a common catalytic subunit that transfers a methyl group from S-adenosyl methionine to the cytosine residue. Among the family members, DNMT3A and DNMT3B serve as de novo methyltransferase that makes new methylation patterns. In contrast, DNMT1 functions as a maintenance methyltransferase that recognizes hemimethylated DNA strands after DNA replication and duplicates the DNA methylation pattern from the parental strand to the daughter strand. ${ }^{20,21}$ However, the DNA methylation of cellular thiol peroxidases is poorly characterized in relation to pathophysiological function.

In this study, we reveal that PrxII expression is silenced by promoter methylation in gastric cancer cells and that PrxII gene silencing is a prerequisite for promoting gastric cancer cell survival and migratory activities. Importantly, our bioinformatics analysis shows that reduced PrxII expression positively correlates with poor survival in gastric cancer patients.

\section{MATERIALS AND METHODS}

\section{Antibodies and reagents}

Polyclonal rabbit antibodies against human PrxI and PrxII were previously described. ${ }^{22}$ The $\alpha$-tubulin antibody was purchased from AbFrontier (Seoul, Korea). Antibodies against p-FAK (Y397), FAK, p-ERK (Thr-202/Tyr-204), p-Src (Y416), pAkt (S473), Akt, caspase-3 and $\beta$-catenin were from Cell Signaling Technology (Danvers, MA, USA). Antibodies against extracellular signal-regulated kinase 2 (ERK2) and c-Src were from Santa Cruz Biotechnology (Dallas, TX, USA). The $\beta$-actin antibody was from AbClon (Seoul, Korea). The E-cadherin antibody was from Millipore (Billerica, MA, USA). Antibodies against occludin and ZO-1 were from Invitrogen (Carlsbad, CA, USA). The tumor necrosis factor-related apoptosis-inducing ligand (TRAIL) was from R\&D Systems (Minneapolis, MN, USA). 5Azacytidine was purchased from Sigma-Aldrich (St Louis, MO, USA). PP2 and PP3 were purchased from Calbiochem (San Diego, CA, USA).

\section{Cell culture}

The SNU16, SNU216, SNU668, MKN28, MKN45, MKN74 and KATOIII human gastric cancer cell lines were provided by the National Cancer Center (Ilsan, Korea). The SNU484 cell line was purchased from the Korean Cell Line Bank (Seoul, Korea). SK4 was obtained from the Department of Systems Biology at the University of Texas MD Anderson Cancer Center (Houston, TX, USA). The Yonsei Cancer Center cell lines were obtained from the Song-Dang Institute for Cancer Research at Yonsei University College of Medicine (Seoul, Korea). All cell lines were cultured with RPMI 1640 medium (Lonza, Walkersville, MD, USA) supplemented with $10 \%$ fetal bovine serum (Welgene Inc, Gyeongsan, Korea), $100 \mathrm{U} \mathrm{ml}^{-1}$ penicillin and $100 \mathrm{U} \mathrm{ml}^{-1}$ streptomycin (Lonza) at $37^{\circ} \mathrm{C}$ in a humidified $5 \% \mathrm{CO}_{2}$ incubator.

\section{Immunoblotting}

Cells were washed with cold phosphate-buffered saline (PBS) and lysed in lysis buffer (20 mm HEPES (pH 7.0), $150 \mathrm{~mm} \mathrm{NaCl}, 10 \%$ glycerol, 1\% Triton X-100, 2 mм EGTA ( $\mathrm{pH} 8.0$ ), 1 mм EDTA, 5 mм $\mathrm{Na}_{3} \mathrm{VO}_{4}, 5 \mathrm{~mm} \mathrm{NaF}, 1 \mathrm{~mm}$ AEBSF, $5 \mu \mathrm{g} \mathrm{ml}^{-1}$ aprotinin and $5 \mu \mathrm{g} \mathrm{ml} l^{-1}$ leupeptin). Lysates were centrifuged at $12000 \mathrm{~g}$ and $4^{\circ} \mathrm{C}$ for $10 \mathrm{~min}$. The supernatants were collected and used for immunoblot analysis. The immunoreactive bands were detected by an enhanced chemiluminescence reagent and quantified with the ImageQuant program.

\section{Quantitative real-time PCR}

Total RNA was extracted from cells using an RNeasy Mini Kit (Qiagen, Hilden, Germany) according to the manufacturer's protocol. cDNA was synthesized from $1 \mu \mathrm{g}$ of total RNA using M-MLV Reverse Transcriptase (Promega, Fitchburg, WI, USA). The cDNA was used as the template for real-time PCR using SYBR Green (Applied Biosystems, Foster, CA, USA). The following primer sequences were used for PCR: forward (5'-CACCTGGCTT-GGATCAACACC- $3^{\prime}$ ) and reverse (5'-CAGCACGCCGTAATCCTCAG-3') for human PrxII; forward $\left(5^{\prime}\right.$ TACCTGGACGAC-CCTGACCTC-3) and reverse (5'-CGTTGCAT CA-AAGATGGACA- $3^{\prime}$ ) for DNMT1; forward (5'-TATTGATGA GCGCACAAGAGAGC- $3^{\prime}$ ) and reverse (5'-GGGTGTTCCAGGGTAA CATTG-AG-3') for DNMT3A; and forward (5'-GGCAAGTTCTCC GAGG-TCTCTG- $\left.3^{\prime}\right)$ and reverse (5'-TGGTACATGGCTTTTCGATAGGA-3') for DNMT3B.

\section{Methylation-specific PCR}

Genomic DNA was extracted from cancer cells using Laird's lysis buffer ( $1 \%$ sodium dodecyl sulfate, $50 \mathrm{~mm}$ Tris ( $\mathrm{pH} 8.0$ ), $100 \mathrm{~mm}$ EDTA, $100 \mathrm{~mm} \mathrm{NaCl}$ and $10 \mu \mathrm{g} \mathrm{ml}^{-1}$ Proteinase K). After shearing, single-stranded DNA (500 ng) was treated with sodium bisulfite using an EZ DNA Methylation Kit (Zymo Research, Irvine, CA, USA) according to the manufacturer's protocol and then subjected to methylation-specific PCR (MSP). The following primers were used for MSP: (forward (5'-TTTTAGGCGGAGTCGTGC-3') and reverse $\left(5^{\prime}\right.$-AAAAACGAACGAACGAAACG- $\left.{ }^{\prime}\right)$ for MSP; and forward $\left(5^{\prime}\right.$ TTTAGGTGGAGTTGTGTGT- $\left.3^{\prime}\right)$ and reverse $\left(5^{\prime}\right.$-ACAAACAAA CAAAACAATCA-3') for non-MSP. DNA from whole blood was used as a control for the demethylated reaction. Water was used as a negative PCR control in both reactions.

\section{siRNA-mediated knockdown}

Small interfering RNA (siRNA) oligonucleotide duplexes were synthesized by Bioneer (Seoul, Korea). The control firefly luciferase siRNA was synthesized by Dharmacon (Lafayette, CO, USA). SiRNA duplexes 
were transfected using Lipofectamine RNAiMAX (Invitrogen) according to the manufacturer's protocol. The following siRNA sequences were used for knockdown: human DNMT1 (5'-GGAAGAAGAGUU ACUAUAA-3'), human DNMT3A (5'-GCACUGAAAUGGAAAG GGU-3'), human DNMT3B (5'-GAAAGUAC-GUCGCUUCUGA-3'), and human c-Src (5'-GUGUCUUAAUACUGUCCUU- $\left.3^{\prime}\right)$.

\section{Retrovirus production}

Retroviruses encoding wild-type (WT) human PrxII and the inactive CS double mutant (Cys51Ser/Cys172Ser) were generated using a bicitronic pLXIN vector and a Retro-XQ vector system (Clonetech, Mountain View, CA, USA). Retroviruses encoding PrxII (WT and CS mutant) were prepared from $293 \mathrm{~T}$ cells by calcium phosphate transfection using $20 \mu \mathrm{g}$ DNA (PrxII-WT, PrxII-CS mutant, VSVG and $\mathrm{Gag} / \mathrm{Pol}$ ) and $2 \times \mathrm{HBS}$ (Hepes Buffered Saline). After $8 \mathrm{~h}$ of incubation at $37^{\circ} \mathrm{C}$, the cells were washed with cold PBS, and fresh culture media were added. After a 24-h transfection, the culture supernatant from the transfected cells was collected, followed by filtration through $0.45 \mu \mathrm{m}$ filter, and the supernatants were stored at $-70^{\circ} \mathrm{C}$. For retroviral infection, the viral aliquot was thawed in a $37^{\circ} \mathrm{C}$ water bath and mixed with $10 \mu \mathrm{g} \mathrm{ml}^{-1}$ polybrene prior to infection.

\section{Colony formation and proliferation assay}

Cells were infected with PrxII-encoding retroviruses for $48 \mathrm{~h}$. Then the cells were trypsinized and counted using a CountessII Automated Cell
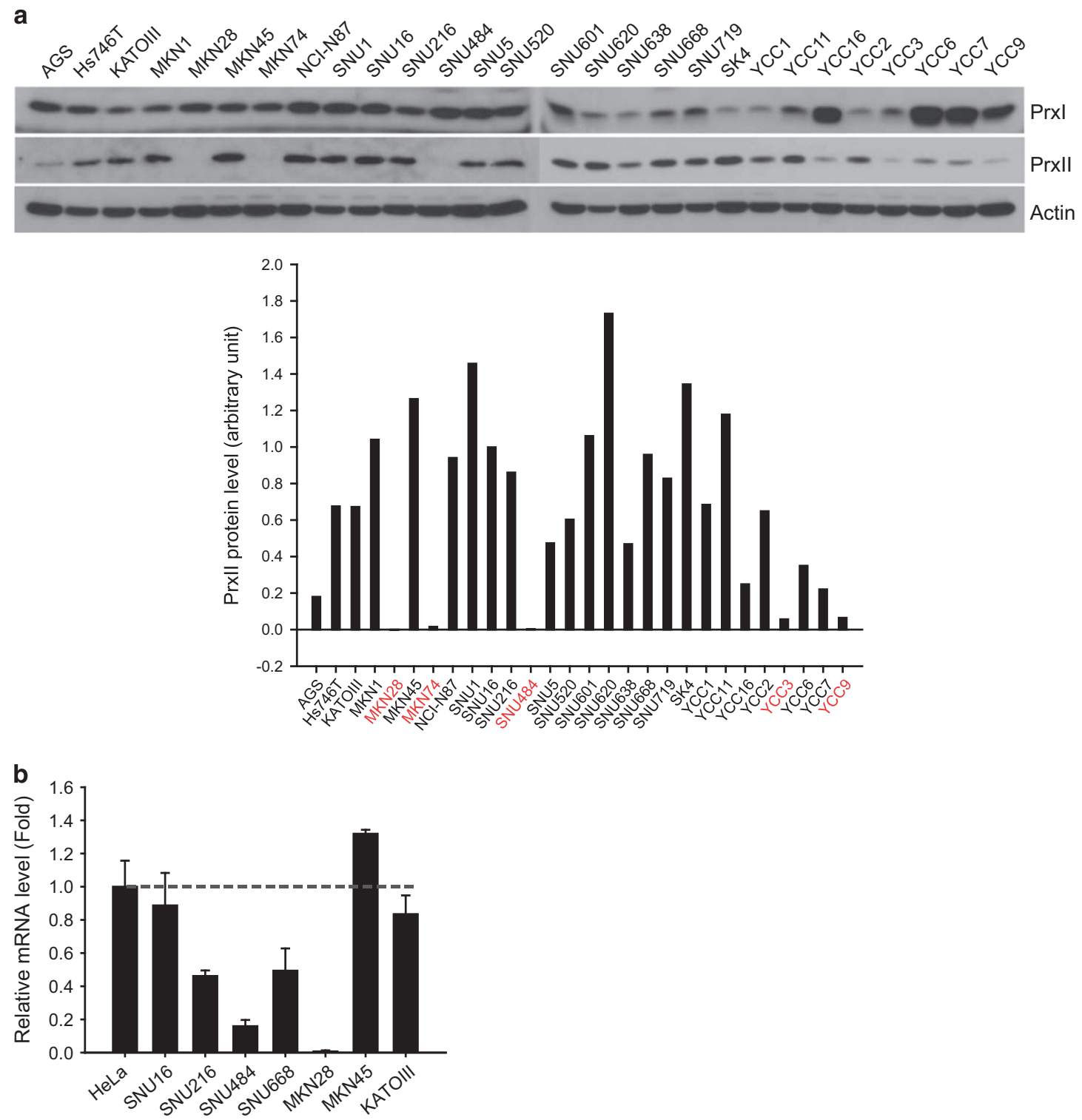

Figure 1 Endogenous Prxll expression in gastric cancer cell lines. (a) Cell lysates from 28 gastric cancer cell lines were prepared and subjected to immunoblot analysis. $\beta$-Actin was used as a loading control. The band intensities of Prxll were quantified using the ImageQuant system and normalized to the $\beta$-actin band. The graph shows the mean relative band intensities from two independent experiments. The cell lines with very low or no Prxll expression are indicated in red. (b) Prxll mRNA levels were determined by quantitative real-time PCR using $\beta$-actin as a reference gene. Prxll mRNA levels in HeLa cells were analyzed as a reference for comparison. RNA was isolated from three independent sets. The graph shows the mean relative fold change \pm s.d. versus Prxll mRNA levels in HeLa cells ( $n=3$ ). 
Counter (ThermoFisher, Waltham, MA, USA). The cells $\left(3 \times 10^{3}\right)$ were plated in $60 \mathrm{~mm}$ culture dishes and grown in fresh media at $37^{\circ}$ $\mathrm{C}$ in a humidified incubator with $5 \% \mathrm{CO}_{2}$ for 10 days. Then the cells were washed with PBS, fixed with $3.7 \%$ paraformaldehyde for $5 \mathrm{~min}$ and stained with $0.05 \%$ crystal violet for $30 \mathrm{~min}$. After washing the cells with cold PBS twice and air-drying for several minutes, the dishes were photographed. The number of colonies was counted using the ImageJ software.

Cell growth was examined using a WST1 assay (Roche Diagnostics, Indianapolis, IN, USA). Retrovirus-infected cells $\left(3 \times 10^{3}\right)$ were seeded in a 96-well culture plate. After infection for the indicated time periods, $10 \mu \mathrm{l}$ of WST1 reagent was added, followed by incubation at $37^{\circ} \mathrm{C}$ incubator for $1 \mathrm{~h}$. The absorbance was measured at $450 \mathrm{~nm}$ using $600 \mathrm{~nm}$ as a reference wavelength with an EPOCH12 microplate reader (BioTek, Winooski, VT, USA) and averaged from triplicate wells. Cell viability was monitored by trypan blue staining. Cells were trypsinized, stained with trypan blue and then counted using a CountessII Automated Cell Counter (ThermoFisher).

\section{Cell death assay}

Cells were washed with cold PBS, trypsinized and collected by centrifugation at 1000 r.p.m. for $3 \mathrm{~min}$. Cells were resuspended in Annexin V-fluorescein isothiocyanate Apoptosis Detection Kit 1 (BD

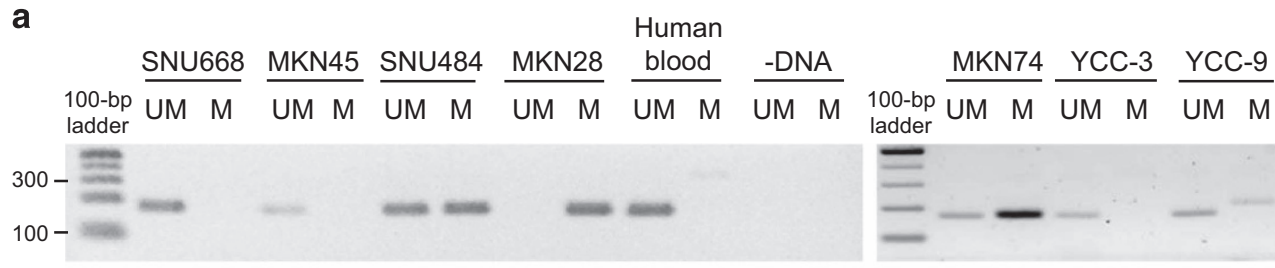

b

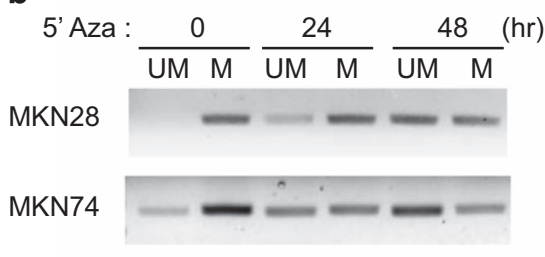

d

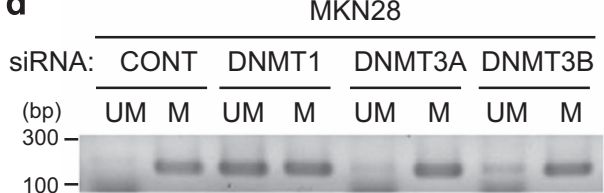

e

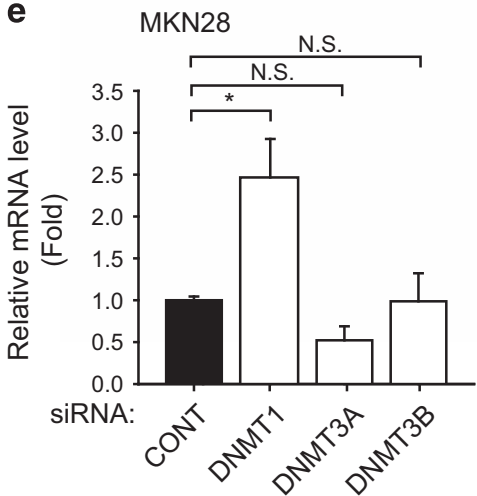

C
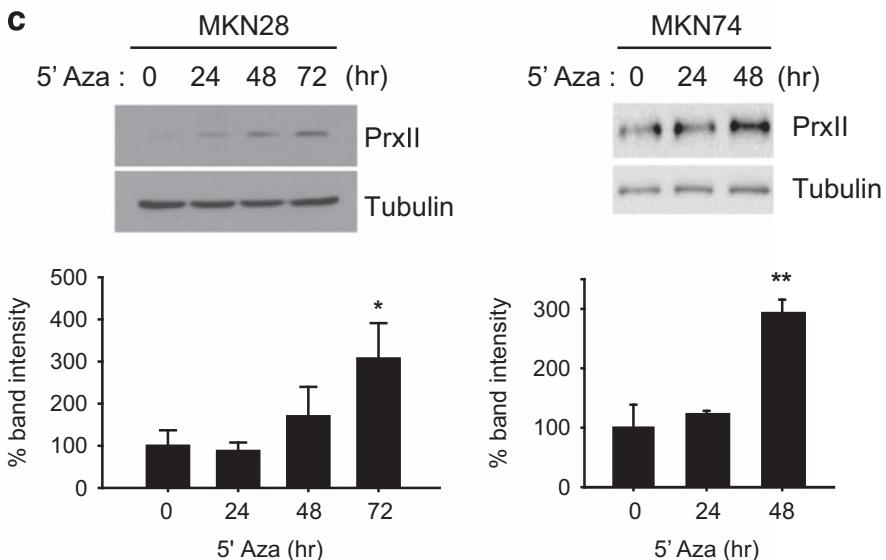

f

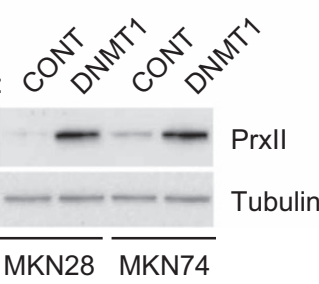

Figure 2 Prxll expression is silenced by promoter methylation. (a) MSP was performed as described in the Material and Methods section. The genomic DNA from human blood tissues was a positive control for the demethylated Prx2 promoter. The template DNA was omitted (-DNA) as a negative control. The unmethylated (UM) and methylated (M) PCR products are 173 and 178 base pairs in size, respectively. An asterisk indicates a non-specific band. A 100-bp DNA ladder was used as a size marker. (b, c) MKN28 and MKN74 cells were treated with $5 \mu \mathrm{m}$ 5-azacytidine (5'Aza) for the indicated times, after which MSP (b) and immunoblot (c) were performed. 5'Aza was refreshed every $24 \mathrm{~h}$. For the immunoblot, the band intensities of Prxll were quantified using the ImageQuant system and normalized to the $\alpha$-tubulin band. The graph shows the mean percentage of band intensity relative to untreated sample $($ lane 1$) \pm \mathrm{s} . \mathrm{d}$. $\left(n=3,{ }^{*} P<0.02\right.$ and $* * P<0.002$ ). (d, e) MKN28 cells were transfected with $100 \mathrm{~nm}$ siRNA against DNMT isotypes twice for a total of 6 days and then subjected to MSP and qRT-PCR analyses. (e) The graph shows the mean relative fold change \pm s.d. of Prxll mRNA level versus the controltransfected cells $\left(n=3,{ }^{*} P<0.05\right)$. NS, not significant. (f) MKN28 and MKN74 cells were transfected with control or DNMT1 siRNAs for 6 days and then subjected to immunoblot analysis. 
Biosciences, San Jose, CA, USA) and incubated at room temperature for $20 \mathrm{~min}$. Cells were treated with propidium iodide $\left(2.5 \mu \mathrm{g} \mathrm{ml}^{-1}\right)$ for $10 \mathrm{~min}$ and then analyzed by flow cytometry using a FACSCalibur system (BD Biosciences). The percentage of dead cells was calculated by subtracting the percentage of unlabeled live cells from $100 \%$ of total cells.

a Retrovirus: $\frac{\text { MKN28 }}{\text { CONT Prxll }} \frac{\text { SNU484 }}{\text { CONT Prxll }}$
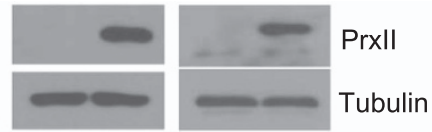

b

Retrovirus:

CONT

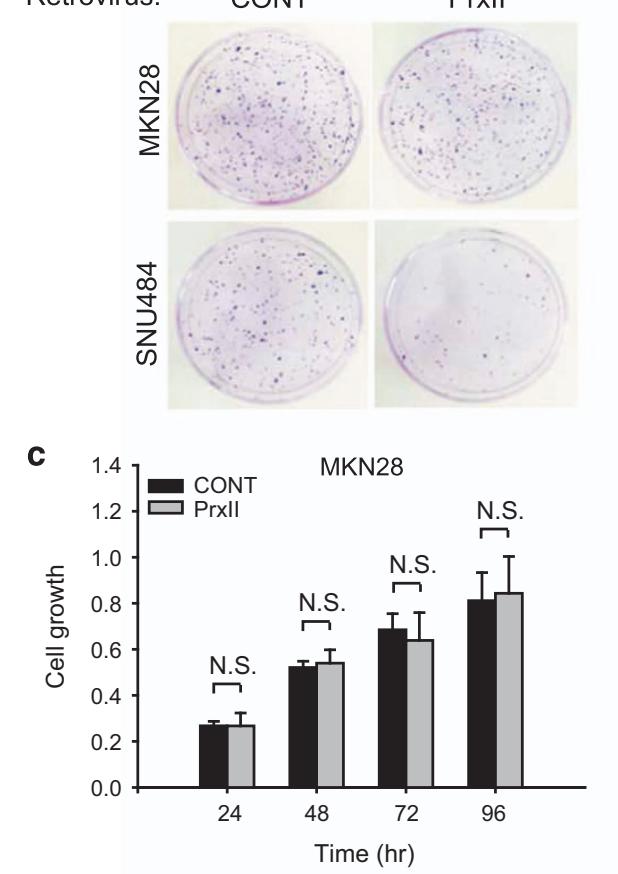

C

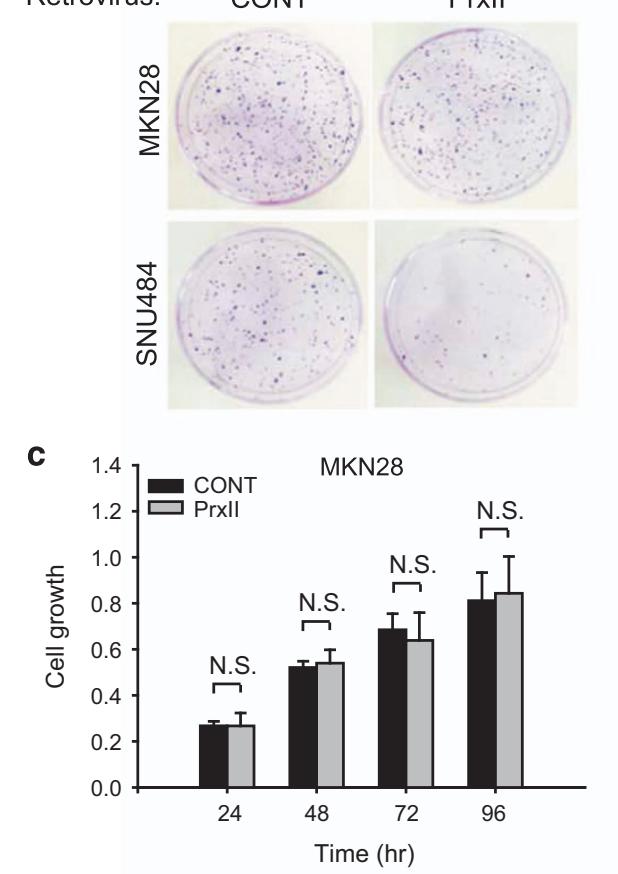

d

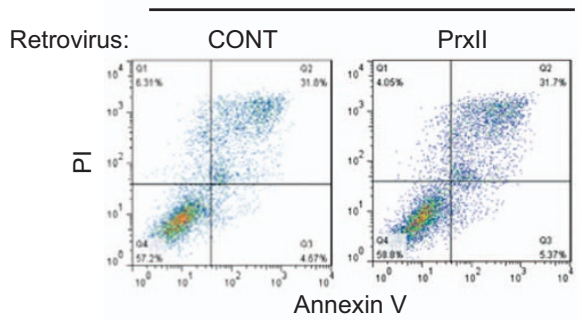

Migration, xCELLigence Real-Time Cell Analyzer and wound-healing assays

Twenty-four-well Transwell culture chambers with polycarbonate membrane inserts with an $8 \mu \mathrm{m}$ pore size (Costar, Washington, DC, USA) were used to conduct the conventional migration assay. The cells were seeded into the upper chamber and stimulated with $20 \%$
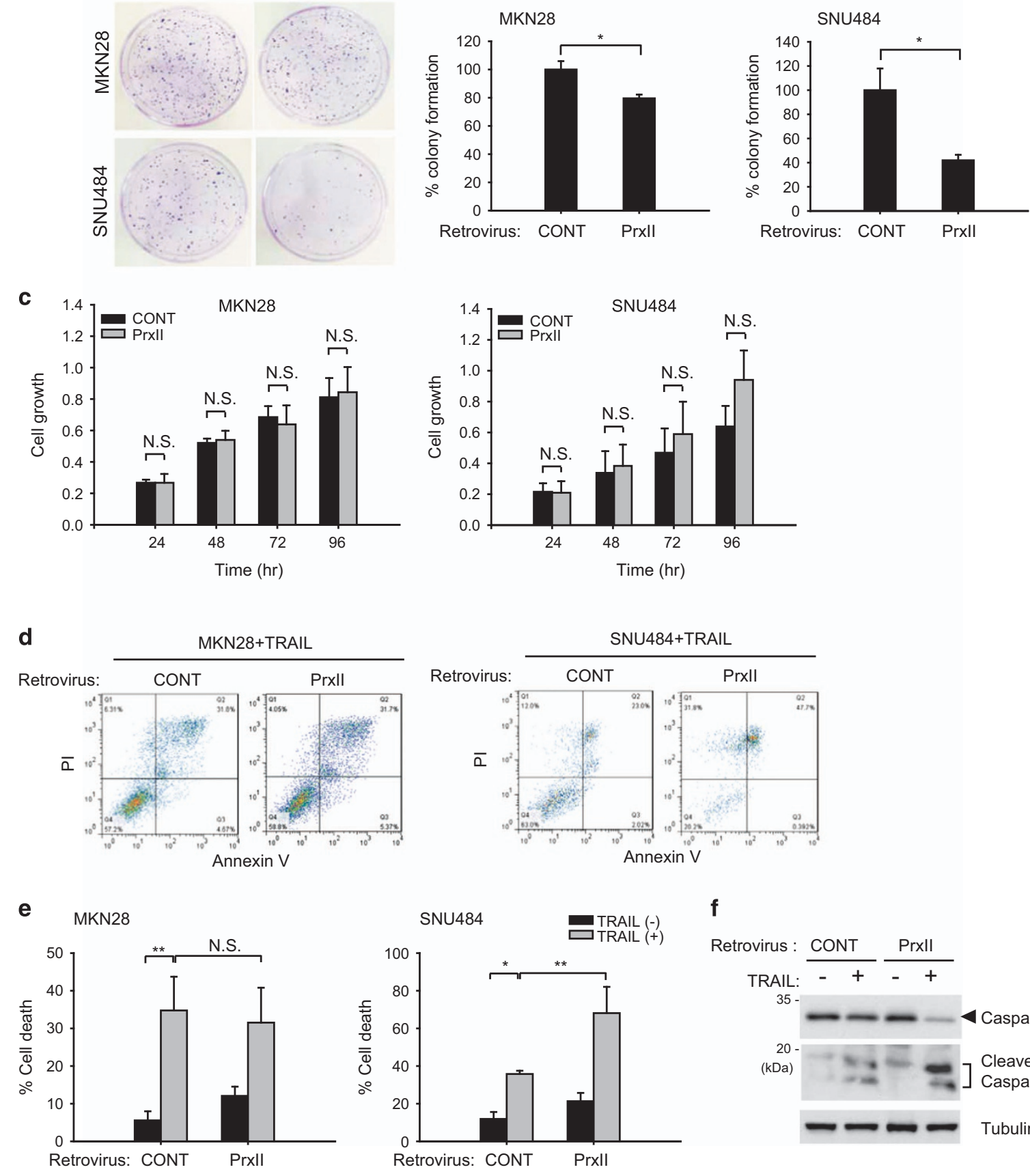

f

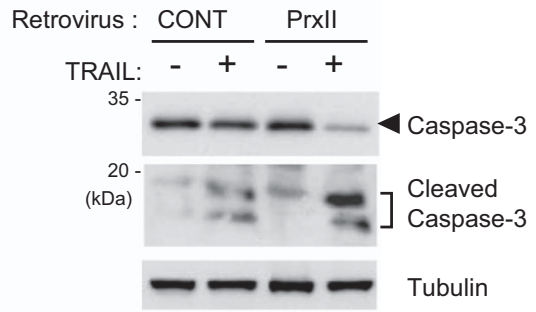


fetal bovine serum. The Transwell chambers were incubated at $37^{\circ} \mathrm{C}$ in a humidified 5\% $\mathrm{CO}_{2}$ incubator. After incubation, the nonmigrated cells were removed from the top of the filter with cotton swabs. Migrated cells that were attached to the bottom side of the filter were fixed with methanol and stained with $0.6 \%$ hematoxylin. The membranes were mounted on the glass slides, photographed and counted. The number of migrated cells was averaged from triplicate sites. Real-time cell migration was measured using an xCELLigence Real-Time Cell Analyzer DP instrument with a CIM-plate 16 (ACEA Biosciences, San Diego, CA, USA).

A wound-healing assay was performed on $35 \mathrm{~mm}$ culture dishes. The monolayer of cells was scratched with a pipette tip. The cells were washed once with PBS and then stimulated with 20\% fetal bovine serum for $24 \mathrm{~h}$. Cell images were taken with an Axiovert200 fluorescence microscope (Zeiss, Oberkochen, Germany). The area of wound closure was measured using the ImageJ software (open source Java image processing program inspired by NIH Image).

\section{Measurement of intracellular $\mathrm{H}_{2} \mathrm{O}_{2}$}

The intracellular $\mathrm{H}_{2} \mathrm{O}_{2}$ level was measured using 5,6-chloromethyl2',7'-dichlorodihydrofluorescein diacetate (CM-DCFH-DA; Invitrogen). The cells were seeded in $35 \mathrm{~mm}$ culture dishes and washed with Hank's Balanced Salt Solution. Then the cells were incubated for 5 min with $10 \mu \mathrm{moll}^{-1}$ CM-DCF-DA. The DCF fluorescence was collected for $20 \mathrm{~s}$ with an inverted Axiovert 200 fluorescence microscope (Zeiss).

\section{Analysis of the TCGA STAD data}

The Cancer Genome Atlas (TCGA) stomach adenocarcinoma (STAD) DNA methylation and mRNA expression data were downloaded at level 3 (that is, as the segmented or interpreted values) from the Broad GDAC Firehose website (released on 28 January 2016). DNA methylation data were obtained for 395 patients using the Illumina Infinium Human Methylation450 or Methylation27 BeadChip technology (San Diego, CA, USA). Transcriptome data were obtained for 415 patients using RNA-Seq experiments on Illumina HiSeq platforms followed by mRNA quantification with the MapSplice-RSEM pipeline. Correlation values between PRDX2 gene DNA methylation and gene expression values were calculated as the Pearson correlation coefficient using an R package. For Kaplan-Meier survival analysis, patients were divided into two groups according to their PRDX2 expression value, and the Gehan-Wilcoxon test was performed to obtain statistical significance.

\section{Statistical analysis}

Data were expressed as the mean \pm s.d. and analyzed using Student's $t$-test in the SigmaPlot 8.0 software (San Jose, CA, USA). P-value was derived to assess statistical significance. $P<0.05$ was considered to be statistically significant.

\section{RESULTS}

PrxII expression is silenced in gastric cancer cells by aberrant promoter methylation

Protein levels of cytosolic 2-Cys Prxs, PrxI and PrxII were examined in 28 gastric cancer cell lines using specific antibodies. Anti-PrxI and anti-PrxII antibodies detected single bands corresponding to the molecular size of PrxI $(22 \mathrm{kDa})$ and PrxII $(21 \mathrm{kDa})$, respectively (Figure 1a). We found that PrxII expression was severely impaired in nine of 28 cell lines, accounting for $32 \%$ by quantification of band intensities. In particular, PrxII expression was completely lost in three cell lines, MKN28, MKN74 and SNU484. By contrast, levels of the closest isoform PrxI protein were similar among the gastric cancer cells tested in parallel. Consistently, quantitative realtime PCR showed that PrxII expression was completely silenced at the transcriptional level in MKN28 cells (Figure 1b). Interestingly, PrxII mRNA expression was slightly present in SNU484 cells. Therefore, the data indicated that both protein and mRNA expression of PrxII were evidently reduced in some gastric cancer cells.

We next investigated the molecular mechanism that resulted in the loss of PrxII expression. Previous studies have shown that $\mathrm{CpG}$ methylation in the PrxII gene promoter was frequently observed in melanoma, leukemia and lymphoma. ${ }^{11,14,15}$ Therefore, we decided to investigate the methylation state of the PrxII promoter region using MSP in seven selected gastric cancer cell lines. The methylation/ demethylation-specific primers targeting the PrxII promoter region were used as previously described ${ }^{9}$ (Supplementary Figure S1). The results showed that the PrxII promoter was methylated in MKN28 and MKN74 cells and only partly methylated in SNU484 cells (Figure 2a). Thus we thought that slight leakage of PrxII transcription in SNU484 cells might be due to the incomplete methylation. Indeed, an immunoreactive band for PrxII protein was weakly detected when the immunoblot was exposed for a long time. To confirm that PrxII silencing resulted from promoter methylation, we treated MKN28 and MKN74 cells with the passive DNA demethylating agent 5'-azacytidine. MSP and immunoblot analysis showed that 5'-azacytidine treatment induced promoter demethylation and subsequent PrxII expression in a timely

Figure 3 Prxll expression promotes the growth of gastric cancer cells. MKN28 and SNU484 cells were infected with control or Prxllexpressing retrovirus for $48 \mathrm{~h}$. (a) Prxll protein levels were assessed by immunoblotting. (b) Retroviral-infected MKN28 and SNU484 cells were grown for 10 days. The colonies were stained and counted. Representative images are shown. The graph shows the mean number of colonies \pm s.d. $\left(n=3,{ }^{*} P<0.01\right)$. (c) Retroviral-infected MKN28 and SNU484 cells were grown in a 96-well culture plate. The cell growth was measured by WST1 at the indicated times. The graph shows the mean absorbance $\left(A_{450}-A_{600}\right) \pm$ s.d. $(n=3)$. NS, not significant. (d, e) Retroviral-infected MKN28 and SNU484 cells were treated with TRAIL (200 $\left.\mathrm{ng} \mathrm{ml}^{-1}\right)$ for 24 h. The cells were collected, stained with propidium iodide (PI) and annexin-V-FITC and then subjected to FACS analysis. (e) The graph shows the mean percentage of dead cells \pm s.d. $\left(n=3,{ }^{*} P<0.05\right.$ and $\left.{ }^{* *} P<0.02\right)$. Representative FACS images are shown. NS, not significant. (f) Retroviral-infected SNU484 cells were treated with or without TRAIL $\left(200 \mathrm{ng} \mathrm{ml}^{-1}\right)$ for $24 \mathrm{~h}$. Cells were lysed for immunoblot analysis using anti-caspase-3 antibody. The immunoblot is a representative of three independent experiments. 
manner (Figures 2b and c). These results indicate that PrxII expression is epigenetically regulated in gastric cancer cells.
To identify the DNMT subtypes responsible for PrxII methylation, we next determined the endogenous mRNA levels of DNMT. The quantitative real-time PCR data revealed that

a
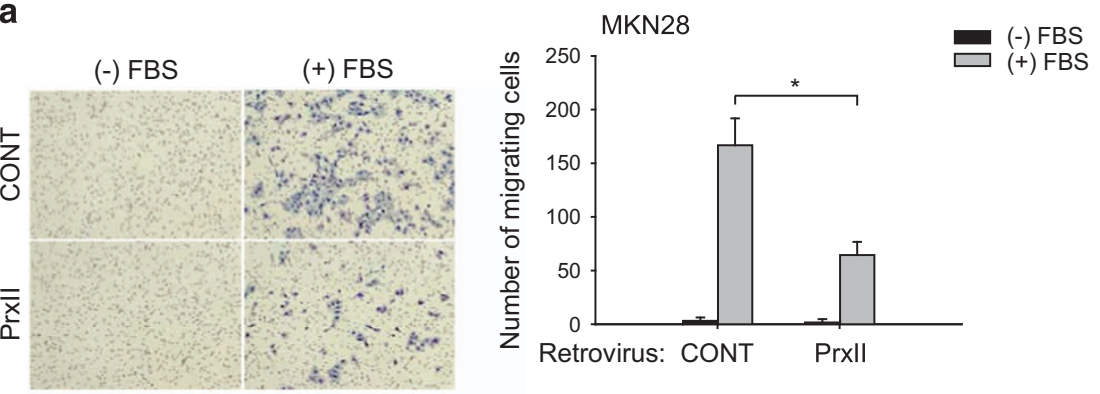

(-) FBS

(+) FBS
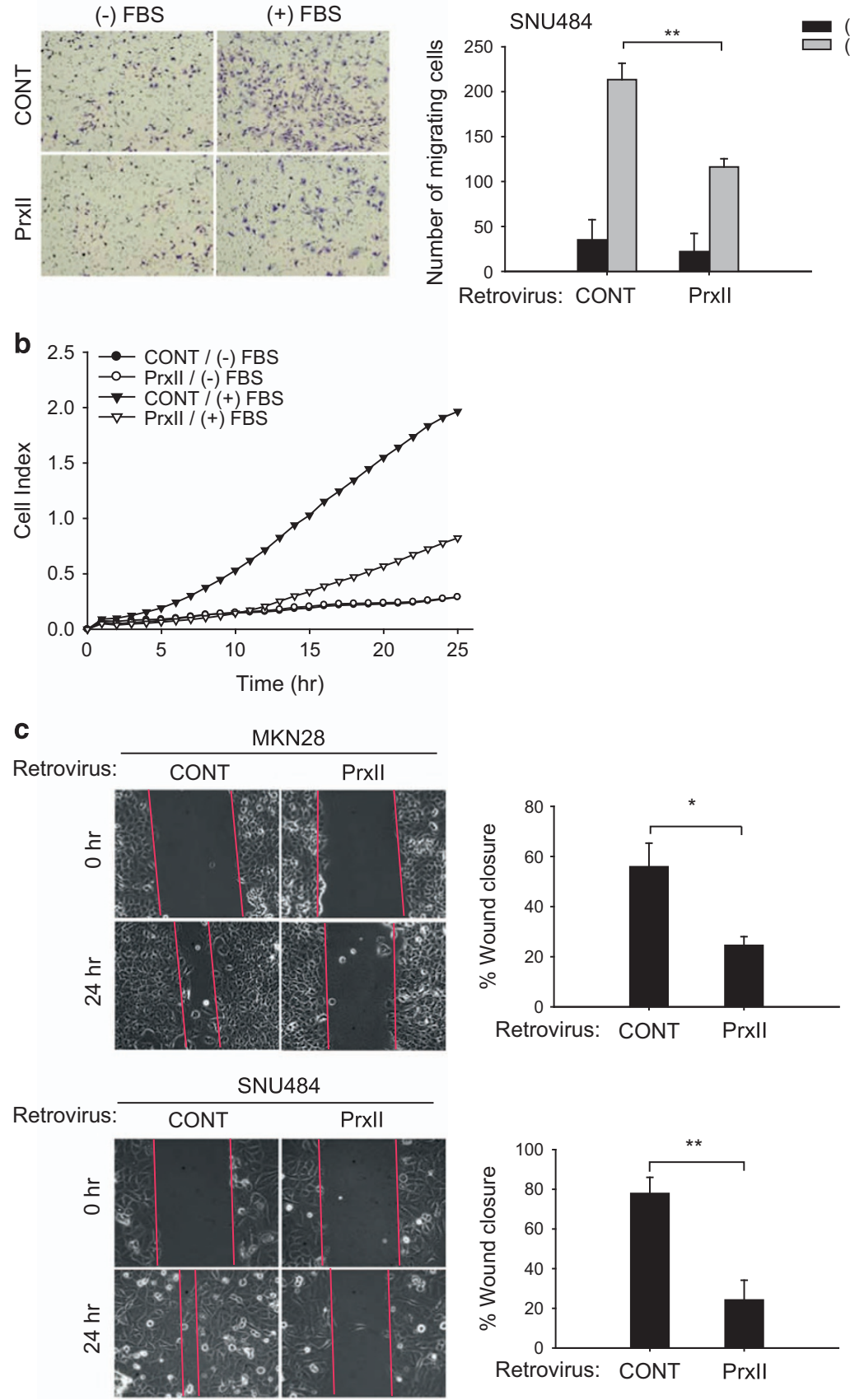
mRNA expression of DNMT1 was 8.5- and 3.7-fold greater than that of DNMT3A and DNMT3B, respectively, in MKN28 cells (Supplementary Figure S2). This result was consistent with a previous study which showed that DNMT1 expression is usually the highest among DNMTs. ${ }^{23}$ We then knocked down DNMTs with specific siRNAs in MKN28 and MKN74 cells. Subsequent MSP showed that demethylation of the PrxII promoter was significantly increased upon DNMT1 knockdown (Figure 2d). Consistently, levels of PrxII mRNA and protein were markedly increased by DNMT1 knockdown (Figures 2e and f). Therefore, we concluded that DNMT1, which is known as the maintenance DNMT, is responsible for PrxII silencing in gastric cancer cells.

\section{PrxII inhibits gastric cancer cell survival}

Numerous studies have indicated a role for PrxII in cell proliferation and migration., ${ }^{9,11,24}$ To examine the cellular function of PrxII in gastric cancer cells, we restored PrxII expression in MKN28 and SNU484 cells using a retroviral system (Figure 3a). A colony-formation assay showed that restored PrxII expression significantly reduced the colonyforming growth of SNU484 and MKN28 gastric cancer cells by $60 \%$ and $20 \%$, respectively (Figure $3 \mathrm{~b}$ ). To examine the reduced colony formation, we subsequently performed cell growth and death analyses. A modified MTT assay using WST1 reagent revealed that PrxII did not affect MKN28 or SNU484 cell growth (Figure 3c). Additionally, trypan blue staining indicated that the cell viability was also unaffected by PrxII expression (Supplementary Figure S3A). However, we noticed that PrxII re-expression appeared to induce a slight increase in apoptotic cells, as indicated by Annexin-V labeling. This notion prompted us to test a potential pro-apoptotic function of PrxII in gastric cancer cells. To this end, we treated the cells with the death inducer TRAIL. Interestingly, PrxII expression greatly sensitized SNU484 gastric cancer cells to TRAIL-induced apoptosis, which subsequently resulted in enhanced caspase-3 activation (Figures 3d-f). In contrast, PrxII expression had no effect on doxorubicin-induced cell death (Supplementary Figure S3B). Collectively, the results suggest that PrxII might be able to suppress gastric cancer cell tumorigenic growth by inducing cell-autonomous apoptosis.

\section{PrxII suppresses gastric cancer cell migratory activities}

The majority of gastric cancer deaths are caused by metastasis. ${ }^{25}$ Therefore, we performed migration assays to examine whether PrxII could regulate gastric cancer cell motility. End-point migration assays were performed using a
Transwell plate with 8 - $\mu$ m pore size inserts. First, we compared the migratory activities among gastric cancer cells with low versus high PrxII expression. The transwell assays demonstrated that MKN28 and SNU484 cells, which had no PrxII expression, exhibited higher migratory activities than MKN45 and SNU668 cells, which had high PrxII expression, respectively (Supplementary Figure S4A). Restored PrxII expression markedly reduced cell migration in response to serum stimulation in both MKN28 and SNU484 cells (Figure 4a). Subsequently, the real-time migration activity of SNU484 cells was examined using the xCELLigence instrument (Figure 4b). Consistent with the end-point migration assay, the slope of the cell index indicated a strong inhibition of cell migration by PrxII expression. Lastly, a wound-healing assay was performed to further confirm the effect of PrxII on gastric cancer cells (Figure 4c). The results showed that the percentage of wound closure in the control cells was greater than that in PrxIIexpressing cells, which supports that gastric cancer cells lacking PrxII migrate faster. Conversely, PrxII knockdown drastically increased serum-induced migration in SNU668 cells (Supplementary Figure S4B). Although there is genetic heterogeneity among gastric cancer cell lines, these data strongly suggest that PrxII is a key factor that regulates gastric cancer cell motility.

We further studied the mechanism by which PrxII activity inhibits migration in gastric cancer cells. When intracellular $\mathrm{H}_{2} \mathrm{O}_{2}$ level was measured using the fluorescent probe CMDCFH-DA by fluorescence microscopy, the expression of WT PrxII reduced cellular $\mathrm{H}_{2} \mathrm{O}_{2}$ levels (Figures $5 \mathrm{a}$ and b), indicating that PrxII is a key $\mathrm{H}_{2} \mathrm{O}_{2}$-eliminating peroxidase in gastric cancer cells. Given that two active-site cysteine residues, Cys51 and Cys172, are required for the peroxidase activity, we generated a retrovirus encoding an inactive PrxII CS mutant where the Cys51 and Cys172 residues are mutated to serine residues to abrogate PrxII peroxidase activity (Figure 5c). Comparison of cell migration between the cells expressing WT and CS mutant PrxII showed that only the WT PrxII, not the CS mutant, inhibited cell migration (Figure 5d). Collectively, the results implicate that PrxII is the first thiol peroxidase that eliminates cellular $\mathrm{H}_{2} \mathrm{O}_{2}$ and suppresses cell migration in gastric cancer cells.

\section{PrxII suppresses Src kinase activation}

Next, we explored the signaling mechanism underlying the PrxII-mediated regulation of migration in gastric cancer cells. Cell migration can be regulated by several mechanisms. We first examined the status of cell-cell adhesion components in

Figure 4 Inhibitory function of Prxll in gastric cancer cell migration. (a) Transwell migration of retroviral-infected MKN28 and SNU484 cells was assayed in the absence and presence of $20 \%$ FBS for 48 and $24 \mathrm{~h}$, respectively, following serum deprivation. The graph shows the mean number of migrated cells \pm s.d. $\left(n=3,{ }^{*} P<0.005\right.$ and $\left.{ }^{* *} P<0.002\right)$. (b) Real-time migration analysis was performed in an xCELLigence analyzer. Serum-starved SNU484 cells were added to the upper chamber in CIM plate 16 and the migration of live cells was monitored for $25 \mathrm{~h}$. (c) A monolayer of retroviral-infected cells was scratched with a pipette tip and stimulated with $20 \%$ FBS. The initial wounded area was measured right after scratching. The graph shows the mean percentage of wound closure $\pm \mathrm{s} . \mathrm{d}$. $\left(n=3,{ }^{*} P<0.01\right.$ and $* * P<0.0001)$. 
MKN28 and SNU484 cells after restoration of PrxII expression. Immunoblot analysis revealed that the levels of the components of adherens junction, namely, E-cadherin and $\beta$-catenin, and of tight junctions, namely, ZO-1 and occludin, were unaffected by
PrxII expression (Figure 6a). We then examined the activation status of the signaling pathways associated with cell migration via phosphorylation-specific immunoblot analysis. Previously, PrxII silencing was shown to upregulate ERK and Src activation
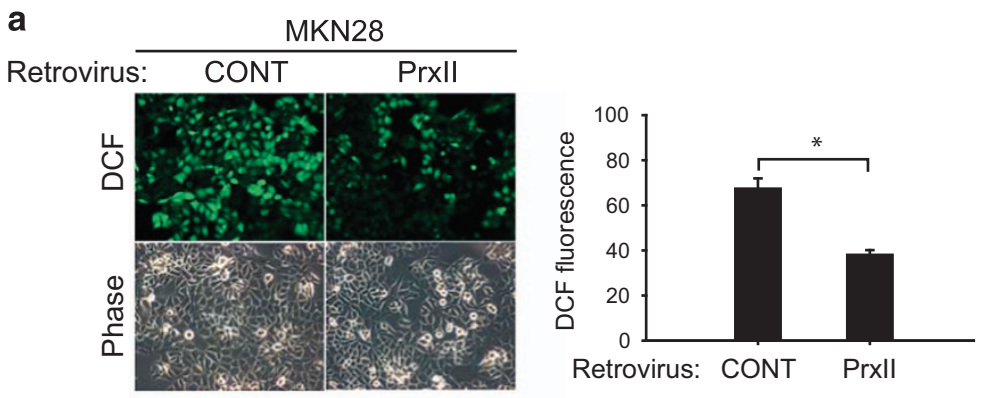

b
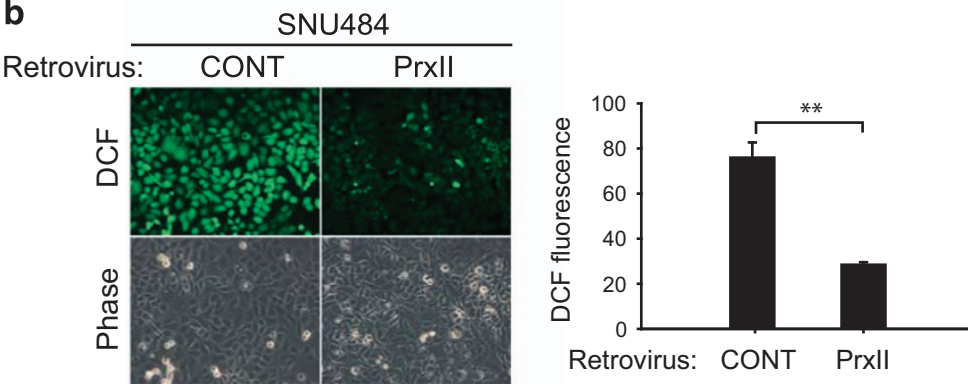

C

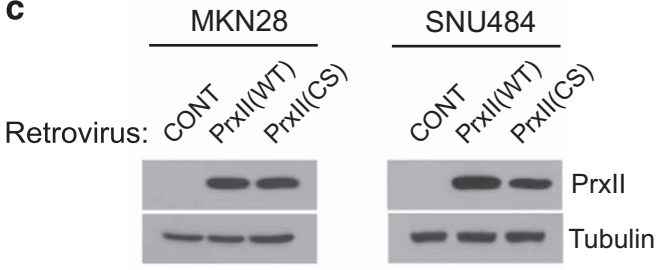

Retrovirus:

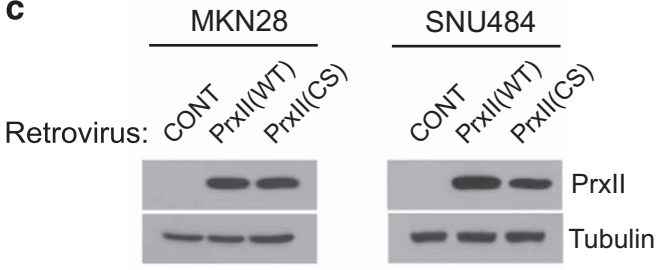

$\mathrm{SNU} 484$

d

MKN28
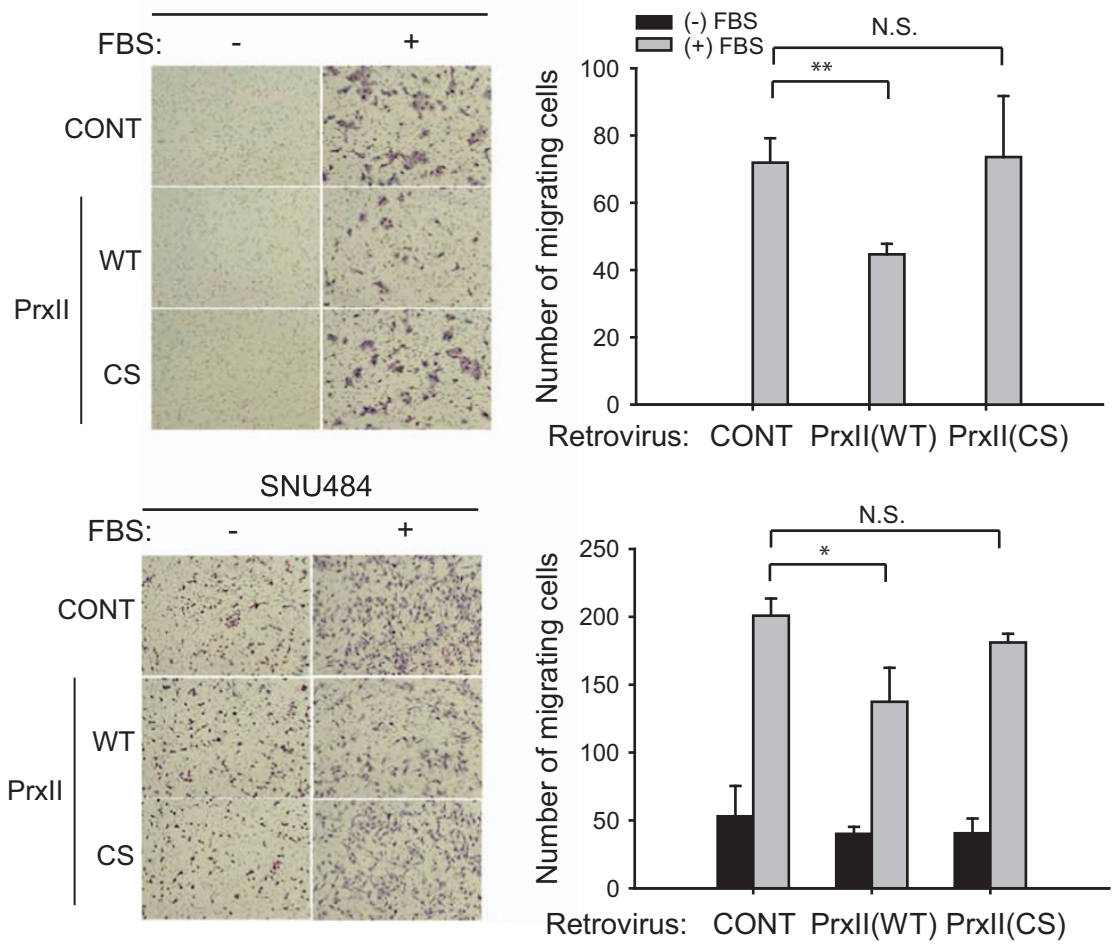
in melanoma cells. ${ }^{9}$ In addition, focal adhesion kinase and Akt are activated by extracellular signals, leading to cell migration and proliferation. Hence, we examined the phosphorylation state of focal adhesion kinase, Src, Akt and ERK in SNU484 and MKN28 cells after the restoration of PrxII expression. It was notable that most kinases were basally activated in both MKN28 and SNU484 cells, even though the cells were grown for $18 \mathrm{~h}$ in serum-deprived conditions (Figure 6b). Exceptionally, the ERK kinase was further activated by serum stimulation in MKN28 cells. Restoration of PrxII expression strongly reduced the basal activation of $\mathrm{c}$-Src kinase but not other kinases in both MKN28 and SNU484 cells (Figure 6c). To confirm that c-Src kinase has an essential role in cell migration, we treated the MKN28 and SNU484 cells with a specific Src inhibitor (PP2). Treatment with the Src inhibitor PP2, not the inactive analog PP3, evidently blocked both MKN28 and SNU484 cell migration, indicating that basal Src activation is essential for gastric cancer cell motility (Figure 6d). In addition, Src knockdown also reduced cell migration in controltransfected MKN28 cells (Figure 6e). However, Src knockdown did not elicit an additional reduction in cell migration in PrxIIoverexpressing MKN28 cells. Thus we confirmed that c-Src activation is a key mediator of PrxII-dependent cell migration in gastric cancer cells.

\section{PrxII expression increases the survival of gastric cancer patients}

To seek a clinical relevance for PrxII in the survival of gastric cancer patients, we analyzed the RNA-Seq data from patients with STAD in TCGA. Bioinformatics analysis revealed that PrxII expression levels in tumors from gastric cancer patients $(n=415)$ was significantly lower than that in normal tissues $(n=35)$ (Figure 7a). Likewise, when PrxII expression was compared between matched tumor and normal samples from 31 individuals, PrxII expression was significantly lower in the tumor tissues (Figure 7b). More importantly, an inverse correlation was observed between PrxII expression and DNA methylation in tumor tissues (Figure 7c), which was consistent with our results using the gastric cancer cell lines MKN28 and SNU484. Finally, Kaplan-Meier survival analysis of gastric cancer patients $(n=408)$ showed that the patient group with high PrxII expression exhibited better survival than the patient group with low PrxII expression (Figure 7d and Supplementary Figure S5). These data firmly indicate that PrxII contributes to gastric cancer patient survival and indicate the role of small molecules that mimic PrxII activity as a novel therapeutic tool for treating gastric cancer patients with silenced PrxII expression.

\section{DISCUSSION}

Gastric cancer is distinctive in terms of tumor heterogeneity, which makes it difficult to predict recurrence and metastasis. ${ }^{3}$ It commonly metastasizes to the liver, peritoneum, lung and bone. The median survival time of patients with metastatic gastric cancer does not exceed 6 months, and the survival period is shorter for patients who are aged $\geqslant 60$ years. ${ }^{26}$ Currently, there are no effective therapeutics available on the drug market for inhibiting gastric cancer metastasis.

It has been well proven that cancer cells exhibit higher ROS levels than normal healthy cells. In other words, they are more susceptible to elevated ROS, which leads to cell death; thus the expression of endogenous antioxidant enzymes is unusually high. ${ }^{4}$ Nonetheless, it is noteworthy that PrxII expression has often been observed to be silenced via CpG methylation in some cancers. ${ }^{11,14,15}$ As an example, we have shown that PrxII silencing in melanoma confers a metastatic benefit by promoting cell migration. ${ }^{9}$ In this study, we showed for the first time that the PrxII promoter is selectively methylated in some gastric cancer cell types. Although the underlying mechanism for the initiation of DNA methylation at the PrxII promoter has yet to be fully elucidated, we found that DNMT1 is responsible for the CpG methylation in the PrxII promoter region that regulates PrxII expression. Given that PrxII is a major ROS scavenger in cancer cells, downregulation of its expression is likely to be the main cause for the elevation in ROS levels. Because ROS are known to upregulate DNMT expression and activity, ${ }^{27}$ we propose a positive feed-forward regulatory loop where high ROS levels due to the silencing of PrxII could affect the DNMT status in cells, which may in turn elicit active gene silencing in gastric cancer cells.

Interestingly, the profound effect of PrxII silencing is the promotion of gastric cancer cell migration. As an underlying mechanism, we found that re-expression of PrxII markedly suppressed c-Src activation. It has been established that most gastric cancer cells are known to have highly activated c-Src, ${ }^{28}$ and elevated c-Src activation is crucial for tumorigenesis by promoting cell proliferation and migration. ${ }^{29}$ Src activation affects cell migration by regulating cell-cell or cell-matrix contacts, such as adherens junctions, tight junctions and focal adhesion. ${ }^{30}$ Therefore, our finding implicates the PrxII gene or its activity mimetic as a therapeutic tool for the prevention of gastric cancer metastasis. Similarly, we have shown that PrxII expression suppresses ERK and c-Src activation in melanoma, ${ }^{9}$

Figure 5 Intracellular $\mathrm{H}_{2} \mathrm{O}_{2}$ elimination by Prxll inhibits the migration of gastric cancer cells. (a, b) Intracellular $\mathrm{H}_{2} \mathrm{O}_{2}$ levels were measured in retroviral-infected MKN28 and SNU484 cells with a $\mathrm{H}_{2} \mathrm{O}_{2}$-sensing fluorescent probe, CM-DCFH-DA. Representative fluorescence (DCF) and phase-contrast images were photographed with a fluorescence microscope, and the fluorescence intensities were quantified by the ImageJ software. The graph shows the mean relative fluorescence \pm s.d. $\left(n=3,{ }^{*} P<0.001\right.$ and $\left.{ }^{* *} P<0.0005\right)$. (c) Prxll wild-type (WT) and inactive mutant (CS) were retrovirally expressed in MKN28 and SNU484 cells. (d) Transwell migration of retroviralinfected and serum-starved MKN28 and SNU484 cells was assayed in the absence and presence of $20 \%$ FBS for 24 and $48 \mathrm{~h}$, respectively. The graph shows the mean number of migrated cells \pm s.d. $\left(n=3,{ }^{*} P<0.02\right.$ and $\left.{ }^{* *} P<0.001\right)$. 
a

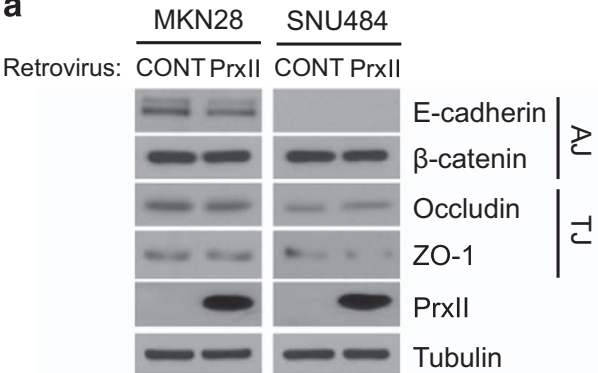

b

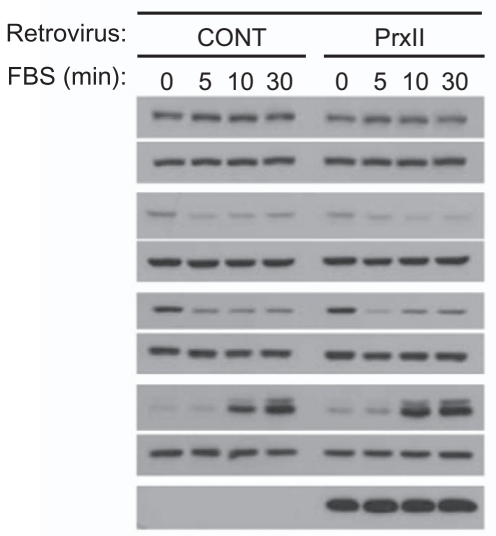

SNU484

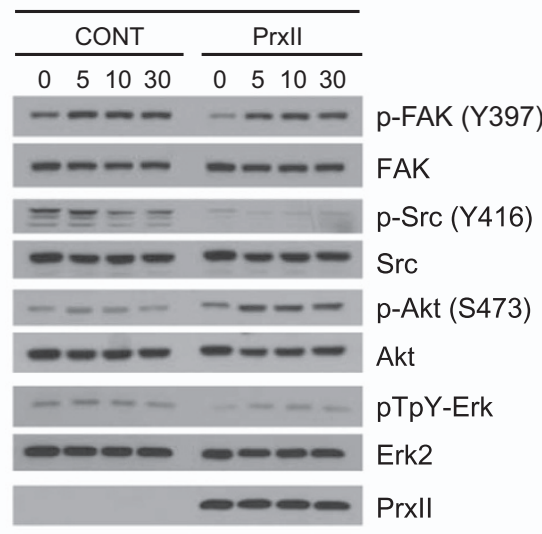

c
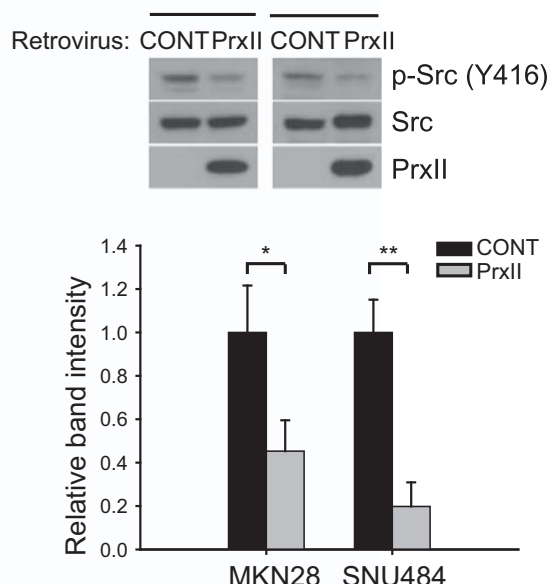

d (-) FBS

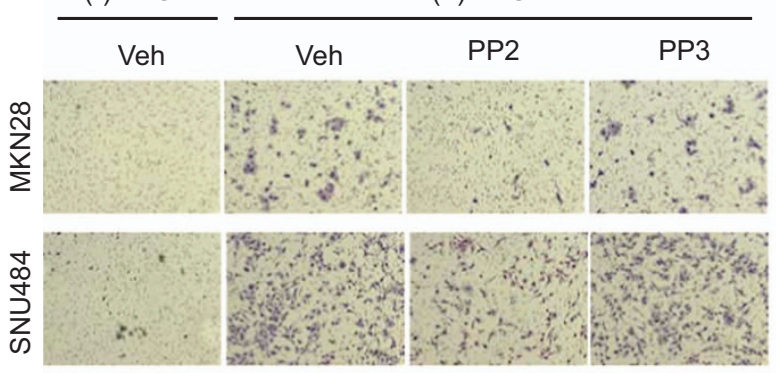

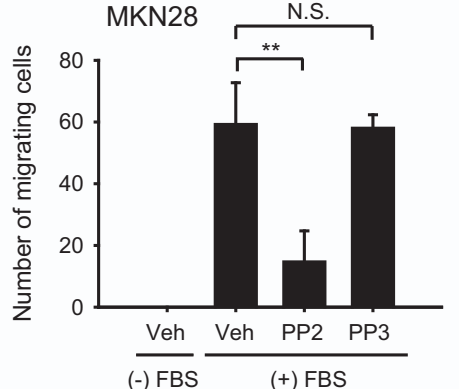

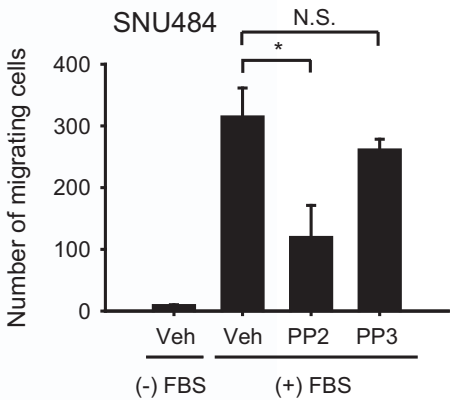

\section{e}

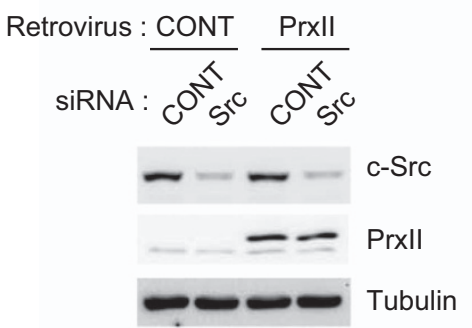

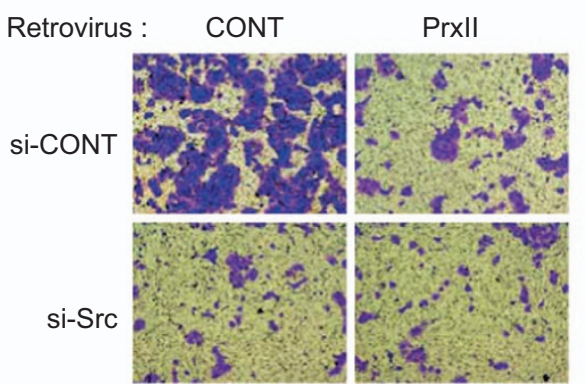

Figure 6 Prxll expression inhibits Src kinase activation. (a-c) Retroviral-infected MKN28 and SNU484 cells were analyzed by immunoblotting for the indicated proteins. Cells were serum starved for $18 \mathrm{~h}$ prior to FBS stimulation. In (c), the intensities of the p-Src bands were quantified using the ImageQuant system and normalized to the Src band. The graph shows the mean relative band intensities versus the control sample \pm s.d. $\left(n=3,{ }^{*} P<0.02\right.$ and $\left.{ }^{* *} P<0.005\right)$. (d) Transwell migration of MKN28 and SNU484 cells was measured in the presence and absence of Src inhibitor (PP2). PP3 was used as a control inactive analog of PP2. The graph shows the mean number of migrated cells \pm s.d. $\left(n=3,{ }^{*} P<0.01\right.$ and $\left.* * P<0.001\right)$. (e) Retrovirus-infected MNK28 cells were transfected with control or c-Src siRNAs for an additional $24 \mathrm{~h}$ and then subjected to Transwell migration assay. The graph shows the mean number of migrated cells $\pm \mathrm{s}$.d. $\left(n=3,{ }^{*} P<0.002\right)$. A representative immunoblot shows the Prxll and $c$-Src protein levels. NS, not significant. 

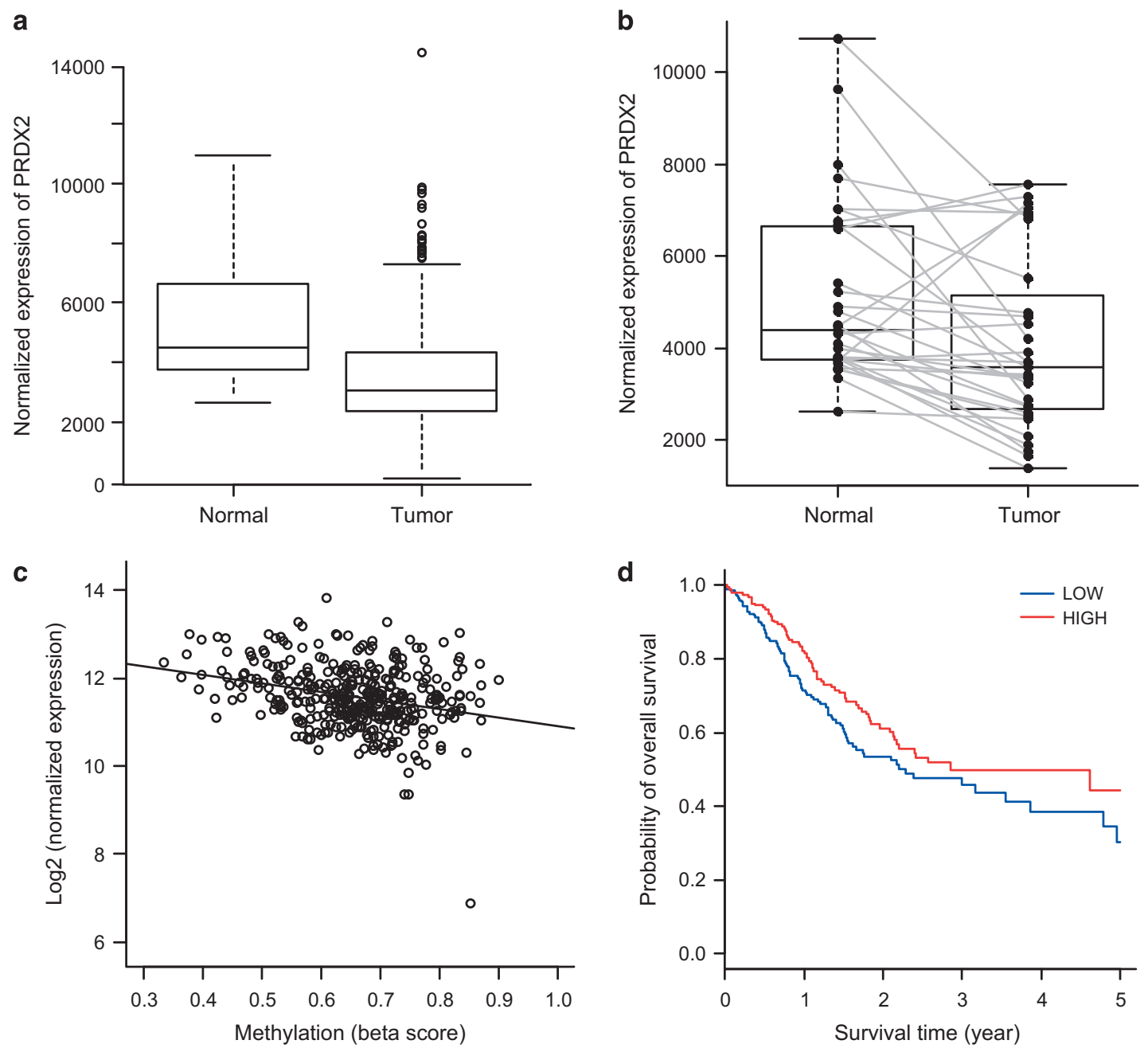

Figure 7 Gene expression, methylation and survival analyses for the PRDX2 gene using the TCGA data. (a) PRDX2 mRNA expression from the TCGA STAD data set, including 35 normal and 415 tumor tissues. PRDX2 expression was significantly decreased in tumors $(P=1.89$ e-05). (b) Comparison of PRDX2 expression in 31 tumor tissues using matched normal tissues as control samples $(P=3.39 \mathrm{e}-03)$. (c) Correlation between mRNA expression and DNA methylation level in 371 tumor tissues (correlation coefficient $=-0.285$, $P=2.27$ e-08). (d) Kaplan-Meier survival plot of the 408 patients divided into two groups based on PRDX2 expression levels ( $P$-value for the Gehan-Wilcoxon test $=0.0422$ ).

where reduced c-Src activation leads to recovery of adherens junctions through reduced $\beta$-catenin phosphorylation on tyrosine 654. Thus it is likely that PrxII-dependent Src regulation is a general mechanism for cancer cell migration and metastasis.

Another unexpected result is that the re-expression of PrxII causes poor colony-forming growth in MKN28 and SNU484 gastric cancer cells. However, this fits well with the poor survival rate of gastric cancer patients with low PrxII expression obtained from TCGA STAD data. The main reason for this finding might be the pro-apoptotic function of PrxII in gastric cancer cells, as evidenced by the data showing that caspase- 3 activation and apoptosis in response to TRAIL treatment was augmented by PrxII expression. In other words, PrxII silencing is a necessary factor for the survival of gastric cancer cells. One possible mechanism involved is the downregulation of Src activation by PrxII expression. Src activation is also known to be a critical step upstream of cell survival pathways, such as the Ras/Raf/ERK pathway and phosphatidylinositol 3-kinase and protein kinase B (Akt) pathways, in cancer cells. ${ }^{31}$ Given that $\mathrm{H}_{2} \mathrm{O}_{2}$ is a second messenger in growth factor signaling, ${ }^{6}$ it is also possible that intracellular $\mathrm{H}_{2} \mathrm{O}_{2}$ levels, which are reduced by PrxII expression, might have a critical proliferative role in gastric cancer cells. In fact, we have shown that PrxII expression negatively regulates platelet-derived growth factor signaling in vascular smooth muscle cells. ${ }^{22}$

In summary, this study reveals novel roles for PrxII in migration inhibition and apoptosis promotion in metastatic gastric cancer. Therefore, PrxII or its activity mimetic could be a strong therapeutic tool for the prevention and eradication of metastatic gastric cancer.

\section{CONFLICT OF INTEREST}

The authors declare no conflict of interest. 


\section{ACKNOWLEDGEMENTS}

We thank Dr Eun-Mi Park, Dr Inho Jo and Dr Dae-Kee Lee for sharing valuable reagents. This study was supported by grants from the National Research Foundation of Korea (2014R1A2A1A01006934, 2012M3A9C5048709 and 2012R1A5A1048236) and from the National R\&D Program for Cancer Control (1420280).

Author contributions: SHH, CM, YJ, DJL, SHK and JHP performed the experiments. SHH, CM, YJ, JHJ, SL, YJP and SWK designed the experiments and interpreted the data. S-YK and SWK conceived the project. SWK supervised the study and wrote the manuscript.

\section{PUBLISHER'S NOTE}

Springer Nature remains neutral with regard to jurisdictional claims in published maps and institutional affiliations.

1 Herrero R, Parsonnet J, Greenberg ER. Prevention of gastric cancer. JAMA 2014; 312: 1197-1198.

2 Siegel RL, Miller KD, Jemal A. Cancer statistics. CA Cancer J Clin 2016; 66: 7-30.

3 Cristescu R, Lee J, Nebozhyn M, Kim KM, Ting JC, Wong SS et al. Molecular analysis of gastric cancer identifies subtypes associated with distinct clinical outcomes. Nat Med 2015; 21: 449-456.

4 Trachootham D, Alexandre J, Huang P. Targeting cancer cells by ROSmediated mechanisms: a radical therapeutic approach? Nat Rev Drug Discov 2009; 8: 579-591.

5 Karisch R, Fernandez M, Taylor P, Virtanen C, St-Germain JR, Jin LL et al. Global proteomic assessment of the classical protein-tyrosine phosphatome and "Redoxome". Cell 2011; 146: 826-840.

6 Rhee SG. Cell signaling. $\mathrm{H}_{2} \mathrm{O}_{2}$, a necessary evil for cell signaling. Science 2006; 312: 1882-1883.

$7 \mathrm{Kim} \mathrm{K}$, Kim IH, Lee KY, Rhee SG, Stadtman ER. The isolation and purification of a specific "protector" protein which inhibits enzyme inactivation by a thiol/Fe(III)/O $\mathrm{O}_{2}$ mixed-function oxidation system. J Biol Chem 1988; 263: 4704-4711.

8 Chae HZ, Chung SJ, Rhee SG. Thioredoxin-dependent peroxide reductase from yeast. J Biol Chem 1994; 269: 27670-27678.

9 Lee DJ, Kang DH, Choi M, Choi YJ, Lee JY, Park JH et al. Peroxiredoxin-2 represses melanoma metastasis by increasing E-Cadherin/beta-Catenin complexes in adherens junctions. Cancer Res 2013; 73: 4744-4757.

10 Zhou S, Han Q, Wang R, Li X, Wang Q, Wang H et al. PRDX2 protects hepatocellular carcinoma SMMC-7721 cells from oxidative stress. Oncol Lett 2016; 12: 2217-2221.

11 Agrawal-Singh S, Isken F, Agelopoulos K, Klein HU, Thoennissen $\mathrm{NH}$, Koehler $\mathrm{G}$ et al. Genome-wide analysis of histone H3 acetylation patterns in AML identifies PRDX2 as an epigenetically silenced tumor suppressor gene. Blood 2012; 119: 2346-2357.

12 Rhee SG, Chae HZ, Kim K. Peroxiredoxins: a historical overview and speculative preview of novel mechanisms and emerging concepts in cell signaling. Free Radic Biol Med 2005; 38: 1543-1552.

$13 \mathrm{Kim}$ YJ, Ahn JY, Liang P, Ip C, Zhang Y, Park YM. Human prx1 gene is a target of Nrf2 and is up-regulated by hypoxia/reoxygenation: implication to tumor biology. Cancer Res 2007; 67: 546-554.

14 Schneider M, Szaumkessel M, Richter J, Ammerpohl O, Hansmann ML, Kuppers $\mathrm{R}$ et al. The PRDX2 gene is transcriptionally silenced and de novo methylated in Hodgkin and Reed-Sternberg cells of classical Hodgkin lymphoma. Blood 2014; 123: 3672-3674.

15 Furuta J, Nobeyama Y, Umebayashi Y, Otsuka F, Kikuchi K, Ushijima T. Silencing of Peroxiredoxin 2 and aberrant methylation of $33 \mathrm{CpG}$ islands in putative promoter regions in human malignant melanomas. Cancer Res 2006; 66: 6080-6086.
16 Min SY, Kim HS, Jung EJ, Jung EJ, Jee CD, Kim WH. Prognostic significance of glutathione peroxidase 1 (GPX1) down-regulation and correlation with aberrant promoter methylation in human gastric cancer. Anticancer Res 2012; 32: 3169-3175.

17 Peng DF, Hu TL, Schneider BG, Chen Z, Xu ZK, El-Rifai W. Silencing of glutathione peroxidase 3 through DNA hypermethylation is associated with lymph node metastasis in gastric carcinomas. PLOS ONE 2012; 7: e46214.

18 Kass SU, Pruss D, Wolffe AP. How does DNA methylation repress transcription? Trends Genet 1997; 13: 444-449.

19 Esteller M. CpG island hypermethylation and tumor suppressor genes: a booming present, a brighter future. Oncogene 2002; 21: $5427-5440$

20 Moore LD, Le T, Fan G. DNA methylation and its basic function. Neuropsychopharmacology 2013; 38: 23-38.

21 Yokochi T, Robertson KD. Preferential methylation of unmethylated DNA by Mammalian de novo DNA methyltransferase Dnmt3a. J Biol Chem 2002; 277: 11735-11745.

22 Choi MH, Lee IK, Kim GW, Kim BU, Han YH, Yu DY et al. Regulation of PDGF signalling and vascular remodelling by peroxiredoxin II. Nature 2005; 435: 347-353.

23 Robertson KD. DNA methylation, methyltransferases, and cancer. Oncogene 2001; 20: 3139-3155.

24 Kang SW, Rhee SG, Chang TS, Jeong W, Choi MH. 2-Cys peroxiredoxin function in intracellular signal transduction: therapeutic implications. Trends Mol Med 2005; 11: 571-578.

25 Kusano T, Shiraishi N, Shiroshita H, Etoh T, Inomata M, Kitano S. Poor prognosis of advanced gastric cancer with metastatic suprapancreatic lymph nodes. Ann Surg Oncol 2013; 20: 2290-2295.

26 Riihimaki M, Hemminki A, Sundquist K, Sundquist J, Hemminki K. Metastatic spread in patients with gastric cancer. Oncotarget 2016; 7: 52307-52316.

27 Lim SO, Gu JM, Kim MS, Kim HS, Park YN, Park CK et al. Epigenetic changes induced by reactive oxygen species in hepatocellular carcinoma: methylation of the E-cadherin promoter. Gastroenterology 2008; 135: 2128-2140.2140 e1-8.

28 Okamoto W, Okamoto I, Yoshida T, Okamoto K, Takezawa K, Hatashita E et al. Identification of c-Src as a potential therapeutic target for gastric cancer and of MET activation as a cause of resistance to c-Src inhibition. Mol Cancer Ther 2010; 9: 1188-1197.

29 Parsons SJ, Parsons JT. Src family kinases, key regulators of signal transduction. Oncogene 2004; 23: 7906-7909.

30 Parsons JT, Horwitz AR, Schwartz MA. Cell adhesion: integrating cytoskeletal dynamics and cellular tension. Nat Rev Mol Cell Biol 2010; 11: 633-643.

31 Roskoski R Jr. Src protein-tyrosine kinase structure, mechanism, and small molecule inhibitors. Pharmacol Res 2015; 94: 9-25.

This work is licensed under a Creative Commons

Attribution-NonCommercial-NoDerivs 4.0
International License. The images or other third party material in this article are included in the article's Creative Commons license, unless indicated otherwise in the credit line; if the material is not included under the Creative Commons license, users will need to obtain permission from the license holder to reproduce the material. To view a copy of this license, visit http://creativecommons.org/licenses/by-nc-nd/4.0/

(C) The Author(s) 2018

Supplementary Information accompanies the paper on Experimental \& Molecular Medicine website (http://www.nature.com/emm) 